\title{
Comparison of different approaches of selecting endmembers to classify agricultural land by means of hyperspectral data (DAIS7915)
}

\author{
Kneubühler, Mathias ; Schaepman, Michael E ; Kellenberger, Tobias W
}

\begin{abstract}
A major problem in classification of hyperspectral datasets is the reliable selection of reference spectra to classify heterogeneous vegetated areas. In this work, different approaches of end-member collection for spectral unmixing of hyperspectral data are presented, evaluated and compared to each other. The selected methods include high resolution ground spectroradiometric measurements, a scene based approach and modelling of the end-member spectra using a SVAT (soil-vegetation-atmospheretransfer) approach. The presented methods are discussed and verified with an extensive ground truth collected in the observed area.
\end{abstract}

DOI: https://doi.org/10.1109/IGARSS.1998.699616

Posted at the Zurich Open Repository and Archive, University of Zurich

ZORA URL: https://doi.org/10.5167/uzh-96578

Conference or Workshop Item

Published Version

Originally published at:

Kneubühler, Mathias; Schaepman, Michael E; Kellenberger, Tobias W (1998). Comparison of different approaches of selecting endmembers to classify agricultural land by means of hyperspectral data (DAIS7915). In: IGARSS 1998, Seattle, USA, 6 July 1998 - 10 July 1998. s.n., 888-890.

DOI: https://doi.org/10.1109/IGARSS.1998.699616 


\title{
Comparison of Different Approaches of Selecting Endmembers to Classify Agricultural Land by Means of Hyperspectral Data (DAIS7915)
}

\author{
Mathias Kneubuehler, Michael E. Schaepman, Tobias W. Kellenberger \\ Remote Sensing Laboratories (RSL), Department of Geography, \\ University of Zürich, $\mathrm{CH}-8057$ Zürich, Switzerland \\ Phone: +41163552 46, Fax: +4116356846 \\ mail: kneub@geo.unizh.ch, schaep@geo.unizh.ch, knelle@geo.unizh.ch \\ http: //www.geo.unizh.ch/rsl
}

\begin{abstract}
A major problem in classification of hyperspectral datasets is the reliable selection of reference spectra to classify heterogeneous vegetated areas. In this work, different approaches of endmember collection for spectral unmixing of hyperspectral data are presented, evaluated and compared to each other. The selected methods include high resolution ground spectroradiometric measurements, a scene based approach and modelling of the endmember spectra using a SVAT (soil-vegetation-atmosphere-transfer) approach. The presented methods are discussed and verified with an extensive ground truth collected in the observed area.
\end{abstract}

\section{INTRODUCTION}

In August 1997 the Digital Airborne Imaging Spectrometer (DAIS7915) operated by DLR (German Aerospace Research Establishment) has been flown over an intensively cultivated agricultural area, the Limpach Valley ( $470 \mathrm{~m}$ a.s.l.) located in Western Switzerland. The area covered by the DAIS is $2.5 \times 10 \mathrm{~km}$ and consists mainly of crops, meadows and sugar beet.

The DAIS-7915 is a 79-channel high-resolution optical spectrometer that covers the wavelength-range between $500 \mathrm{~nm}$ and $12000 \mathrm{~nm}$ using a Kennedy type scanning mechanism. The first 72 channels cover the reflective part of the electromagnetic spectrum whereas the channels 73-79 cover the MIR and TIR range. The DAIS is operated aboard DLR's Dornier DO228 aircraft and has a swath angle of $52^{\circ}$, subdivided into 512 pixels per scanline. The flight altitude was around $3840 \mathrm{~m}$ a.s.1, resulting in a ground sampling distance of 5.5 $\mathrm{m}$. The flightline is recorded using a differential GPS on board of the DO228 and on preselected ground control points.

Numerous spectroradiometric measurements on selected reference targets have been taken in the test area using a GER-3700 spectroradiometer. This 704 channel spectroradiometer covers the $400-2500 \mathrm{~nm}$ wavelength range. The GER-3700 is calibrated in the laboratory by RSL [1].

Mapping of the land cover and determination of the field borders, which allows for assessing the quality of the spectral unmixing results, is based on areal photography. This coregistration process helps to identify more than 90 fields with their land cover on the image.

\section{METHODOLOGY}

The DAIS-7915 data are provided as radiance-calibrated data from DLR. The preprocessing of the imaging spectrometer data includes an MNF (Minimum Noise Fraction) transformation of the data to reduce noise and atmospheric correction to convert the data to apparent reflectances. Atmospheric correction is performed using ATCOR2 [2], an atmospheric correction program, based on lookup tables generated with a radiative transfer code (MODTRAN-3). The composition of the atmosphere is characterized using water vapor content, aerosol type, optical thickness (visibility in $\mathrm{km}$ ) and the solar zenith angle.

Since no in-situ meteorological data is available for the time of the datatake, the horizontal visibility is determined using a comparison of different modelling approaches with in-situ reference spectra. This procedure is carried out iteratively, based on IFCALI [2]. The best correlation with reference measurements is found with an atmosphere based on midlatitude summer conditions, rural aerosol-type and a visibility of $25 \mathrm{~km}$. Nevertheless, it has to be pointed out, that the atmospheric correction strongly overestimates the water vapor content, resulting in an overcorrection in the $940 \mathrm{~nm}$ water vapor absorption band.

Because of noisy SWIR bands and the importance of specific vegetation related absorption bands [3] (e.g. chlorophyll a and b, water absorption) or the position of the red-edge, the analysis focuses on the first 40 channels of the DAIS. Because of the overcorrection of water vapor absorption, the channels around the $940 \mathrm{~nm}$ water vapor absorption band are also excluded.

Major attention is given to agricultural land, so that four types of linear contributing endmembers are assumed to be present in the area under investigation. They are: dry biomass (ripe wheat), soil, shade and vegetation (grass and sugar beet). The three different approaches for the endmember collection applied are:

$$
\begin{aligned}
& \text { - } \quad \text { image based selection using the inner part of a field } \\
& \text { - } \\
& \text { Spectroradiometric measurements of reference targets }
\end{aligned}
$$

Image based selection: endmembers are selected within the inner part of the field to avoid the selection of adjacency influenced border pixels.

Spectroradiometric measurements of reference targets: reference measurements of reference fields are convolved to the required wavelength of the DAIS-7915 channels.

Modelling endmember spectra: endmembers of green vegetation (grass and sugar beet) are modeled using a SVAT approach. In order to simulate canopy reflectance, a single leaf reflectance model (PROSPECT [4]) and a canopy reflectance model (SAIL [5]) are combined. PROSPECT uses as input a leaf mesophyll structure parameter $\mathrm{N}$, chlorophyll content, leaf dry mass and leaf water content. It simulates leaf reflectance and leaf transmittance between 400 $\mathrm{nm}$ and $2500 \mathrm{~nm}$ as a function of the abovementioned leaf properties. 
The output of the PROSPECT model is then used as input to the SAIL model. The SAIL model uses as input canopy variables (e.g. Leaf Area Index, Leaf Angle Distribution, leaf reflectance and transmittance), soil reffectance, the ratio between diffuse and direct irradiation and solar/view geometry (solar zenith angle, zenith view angle and relative angle between sun and view azimuth [6]. The PROSPECT parameters are calculated by inverting an averaged measured high resolution spectrum of vegetation (grass and sugar beet) and by minimizing the RMS-error between modeled and measured spectra. The SAIL model calculates canopy reflectance under different view zenith angles which become important when performing comparisons with sensors having al large scan angle such as the DAIS. Canopy reflectance is dependent on the sensors changing view zenith angle and the changing relative azimuth angle between sun and viewing direction. Since the main area of interest is completely flat and lies within $13^{\circ}$ to each side from nadir view, only nadir-modeled spectra are used for the modelling approach. However, Fig. 1 and Fig. 2 show the variation in canopy reflectance of grass and sugar beet resulting from changing view zenith angles. Since soil and dry biomass (ripe wheat) do not represent vital vegetation, these endmembers are not modeled, but substituted by the field reflectance measurements. Fig. 1, Fig. 2 and Fig. 3 show the endmember spectra for grass, sugar beet, wheat and soil.

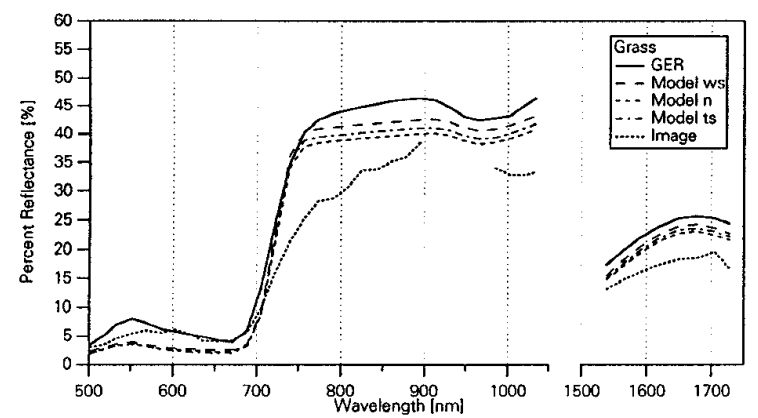

Fig. 1: Measured, image derived and modeled endmember spectra for grass (ws: looking with the sun, n: nadir view, ts: towards the sun)

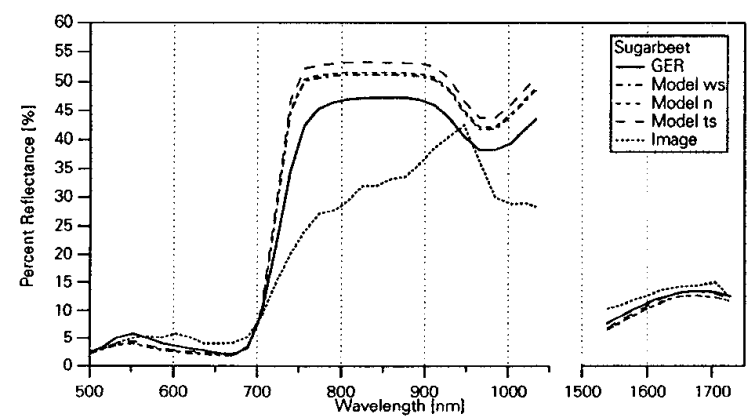

Fig. 2: Measured, image derived and modeled endmember spectra for sugar beet (ws: looking with the sun, $\mathrm{n}$ : nadir view, ts: towards the sun)

The evaluation of the different results of these three approaches of endmember selection for spectral unmixing are discussed focussing on well defined verification areas of sugar beet. Sugar beet is one of the predominant land cover in the observed area. Abundance maps of the sugar beet endmember are generated in a two step approach: First, based on the resulting RMS of the spectral unmixing procedure, which is calculated for each pixel, areas having an RMS larger than (RMS mean +2 Stdev) were excluded from further interpretation. Large RMS values indicate poor unmixing results based on the selected endmembers. Secondly, endmember abundances lower than zero are also excluded. The abundance maps of sugar beet are geocoded using a parametric geocoding approach (PARGE) including a DEM (Digital Elevation Model) and the attitude data of the aircraft [7].

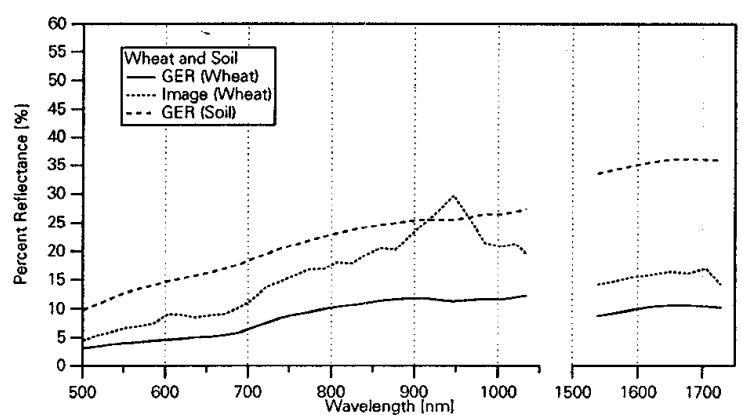

Fig. 3: Measured and image derived endmember spectra for wheat and measured endmember spectrum for soil. The reflectance peak at $940 \mathrm{~nm}$ in the image derived spectrum results from the overcorrection of the water vapor

\section{RESULTS AND CONCLUSIONS}

Spectral mixture analysis is based on the assumption that most of the spectral variation within imaging data is caused by mixtures of a limited number of surface materials. Linear spectral unmixing assumes no interaction between materials and therefore models the observed spectral reflectance as linear combinations of pure endmembers. Each individual spectrum is unmixed, resulting in a dataset of spatial images of endmember abundances [8]. In this work, a weighted constrained linear unmixing is applied, allowing abundances to have values lower than 0 or greater than 1, but summing up to unity [9]. As pointed out by Schanzer [10], abundances can take on values greater than 1 or lower than 0 . If the response of a pixel is purer than the selected endmembers, endmember abundances greater than 1 and lower than 0 will occur.

Fig. 4 shows a geometrically corrected subset of the three unmixing results. The results from image based endmember selection (left), measured endmember spectra (middle) and modeled endmembers (right) are compared to each other focussing on sugar beet. Differences of abundances of the sugar beet endmember on the same fields are obvious for the three approaches. The averaged abundances within three well defined verification areas for sugar beet are 2.03 for image derived endmembers, 0.38 for GER endmembers and 0.14 for modeled endmembers. Image derived endmembers tend to be less pure than measured or modeled endmembers, since they are composed of a mixture of the observed vegetation as well as soil and shade present in the target area. Measuring endmember spectra focussing on the vegetated part itself or modelling them from bio- 
chemical, biophysical and geometrical parameters generally leads to purer spectra of the desired endmember. This fact can be seen in the three images very well. The image based approach (1) shows many fields with some amount of sugar beet abundance whereas no or less abundance of this endmember can be found in the same fields in the measured (2) and modeled (3) endmember approaches. Black fields are sugar beet plantations. They show highest abundances of sugar beet for all three approaches, although the abundance values differ significantly. Pixels as pure as the measured and modeled endmembers are hardly any found. Therefore, the average abundance value for the three verification fields is low.

The scene based approach instead bases on impure endmembers. The field where the endmembers for sugar beet are collected is obviously a mixture of various contributing species like soil, shade, weed and sugar beet. Therefore, evaluation fields with a higher amount of sugar beet lead to a purer response than the endmember itself. This is the case of feasible endmember collection [10]. Abundance values greater than 1 occur. This forces parts of the remaining endmembers to become negative. To solve this problem, an interactive approach of scene based endmember collection is proposed. A first unmixing result allows for identification of purer endmembers than the initial ones. This process can be repeated until a certain "purity-criteria" is reached.

The approach using in-situ reference measurements of endmember spectra and the modelling approach using a SVAT Model may offer the possibility to retrieve biochemical and biophysical parameters from imaging data. Nevertheless, it remains difficult to define a representative endmember spectrum. Averaging multiple measurements of the same target makes an endmember more representative, but unmixing results from measured endmembers varying within 1 standard deviation have shown strong changes in abundances of sugar beet.

Modelling endmember spectra bases on the availability of a range of parameters describing the status of the plant and the architecture of the canopy, which itself is subject to strong BRDF effects. Applications in vegetation studies have to take into account these effects, since airborne imaging spectrometers with large swath angles produce data under strongly differing viewing conditions.

\section{REFERENCES}

[1] SCHAEPMAN, M., SCHLÄPFER, D., MÜLLER, A. AND STROBL, P., 1997: "Ground Spectroradiometric Measurements in Support of the Validation of the Calibration of Digital Airborne Imaging Spectrometer (DAIS 7915) Data", Proc. of the Third International Airborne Remote Sensing Conference and Exhibition, Copenhagen, Vol. 2, pp. 217-223

[2] RICHTER, R., 1996: "Atmospheric correction of DAIS hyperspectral image data", Computers \& Geosciences, Vol. $22,785-793$

[3] VERDEBOUT, J., JACQUEMOUD, S. AND SCHMUCK, G., 1994: "Optical properties of leaves: modelling and experimental studies", in: Imaging Spectroscopy - a Tool for Environmental Observations, eds. Hill, J. and Mégier, J., Euro Courses, Kluwer, Vol. 4, pp. 169-191

[4] JACQUEMOUD, S. AND BARET, F., 1990: "PROSPECT: a model of leaf optical properties spectra", Rem. Sens. Env. 34, 75-91

[5] VERHOEF, W., 1984: "Light scattering by leaf layers with application to canopy reflectance modelling: the SAIL model", Rem. Sens. Env. 16, 125-141

[6] CLEVERS, J.G.P.W., 1994: "Imaging spectrometry in agriculture - plant vitality and yield indicators", in: Imaging Spectroscopy - a Tool for Environmental Observations, eds. Hill, J. and Mégier, J., Euro Courses, Kluwer, Vol. 4, pp. 193 219

[7] SCHLÄPFER, D., MEYER P. AND ITTEN, K.I., 1998: "Parametric Geocoding of AVIRIS Data Using a Ground Control Point Derived Flightpath", Summaries of the Seventh JPL Airborne Earth Science Workshop, (in press), JPL, Pasadena (CA), pp. 6

[8] BOARDMAN, J.W, 1990: "Inversion of high spectral resolution data", Proc. SPIE, Vol. 1298, pp. 222-233

[9] RSI Inc., 1997: “ENVI User's Manual", Vers. 3,0, Lafayette

[10] SCHANZER, D.L., 1993: "Comments on "The Least Squares Mixing Models to Generate Fraction Images Derived for Remote Sensing Multispectral Data'“, TGARS, Vol. 31, p. 747

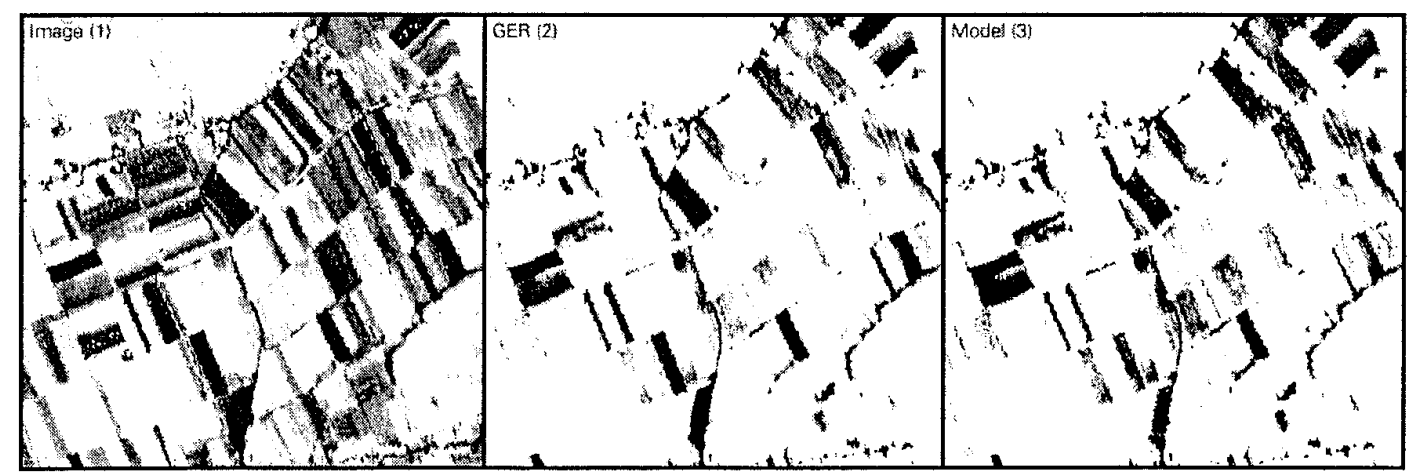

Fig. 4: Abundance maps of sugar beet from image derived (1), in-situ measured (2) and modeled (3) endmember spectra 